\title{
DIELECTRIC DISPERSION IN FERROELECTRIC GLYCINE PHOSPHITE
}

\author{
R. TChUKvinskyi, Z. CZaPla \\ Institute of Experimental Physics, University of Wrocław \\ Pl. M. Borna 9, 50-204 Wrocław, Poland \\ R. Sobiestianskas, A. Brilingas, J. Grigas
}

Faculty of Physics, Vilnius University, Sauletekio Aleja 9, 2054 Vilnius, Lithuania

AND J. BARAN

Institute of Low Temperature and Structure Research, Polish Academy of Sciences Okólna 2, 50-950 Wrocław 2, Poland

(Received April 24, 1997; in final form August 25, 1997)

\begin{abstract}
Dielectric dispersion in ferroelectric hydrogen bonded glicyne phosphite crystal was investigated in the frequency range $100 \mathrm{~Hz}-27 \mathrm{GHz}$. Dielectric relaxation of Debye type observed in the paraelectric phase shows a critical slowing down of the polarization fluctuations. The relaxation frequency decreases with temperature according to $f_{\mathrm{s}}=0.305\left(T-T_{0}\right) \mathrm{GHz}$ in the paraelectric phase. The activation energy for flipping dipole motion $\Delta U=2.07 k T_{c}$ confirms order-disorder character of the phase transition. In the ferroelectric phase pronounced low frequency $(100 \mathrm{~Hz}-1 \mathrm{MHz})$ dispersion related to domain contribution to permittivity was found.
\end{abstract}

PACS numbers: 77.80.Bh

\section{Introduction}

Glycine phosphite Gly $\cdot \mathrm{H}_{3} \mathrm{PO}_{3}$ (abbreviated as GPI) is a newly discovered ferroelectric crystal and undergoes a continuous phase transition at $T_{\mathrm{c}}=224.7 \mathrm{~K}$ [1]. A large anomaly of the dielectric permittivity was found at the Curie point along the $b$-axis of monoclinic symmetry $P 2_{1} / a$. Moreover, a high value of permittivity was observed along the $c^{*}$-axis, as well. It reaches a value of 250 at $T_{\mathrm{c}}$ and decreases slightly on heating to the room temperature. The spontaneous polarization $\left(P_{\mathrm{s}}\right)$ appears along the $b$-axis below $224.7 \mathrm{~K}$. The temperature dependence of $P_{\mathrm{s}}$ is characteristic of the phase transition close to tricritical point. As it results from $\mathrm{X}$-ray investigations [2] the crystal structure is built of infinite chains of hydrogen 
bonded phosphite anions directed along the crystallographic $c$-axis. Two types of symmetrical disordered hydrogen bonds of the length 2.482 and $2.518 \AA$ exist in the room temperature phase. They give the large contribution to the value of the dielectric permittivity along the $c$-axis. However, it seems to be obvious that protons motion along the hydrogen bonds (along the $c$-axis) produces a change of dipole moment in the $b$-axis direction. Thus, most probably, the ordering of the hydrogen bonds along the $c$-axis results in appearance of the spontaneous polarization along the $b$-direction. A similar situation was already found in $\mathrm{KH}_{2} \mathrm{PO}_{4}$-family crystals where the hydrogen bonds are perpendicular to ferroelectric axis [3-4]. Recently, results of dielectric dispersion in the vicinity of $T_{\mathrm{c}} \pm 3 \mathrm{~K}$ were presented both for hydrogenated and deuterated GPI crystals [5, 6].

In this paper we decided to study dynamical dielectric properties along the $b$-axes in the frequency range $100 \mathrm{~Hz}$ to $27 \mathrm{GHz}$ and in the wide temperature range (210-250 K).

\section{Experimental}

The transparent crystals of GPI were grown by a slow evaporation method from saturated water solution of stoichiometric quantities of glycine and phosphorous acid at $301 \mathrm{~K}$. Measurements of the complex dielectric permittivity $\varepsilon^{*}(\omega, T)$ in the frequency range up to $1 \mathrm{MHz}$ were performed by a computer controlled $\mathrm{HP}$ 4284A LCR meter. High-frequency measurements were made by using coaxial and wave guide $[7,8]$ dielectric spectrometers on cooling at a rate of $0.15 \mathrm{~K} / \mathrm{min}$. The samples were coated with gold electrodes.

\section{Result and discussion}

Temperature dependences of the dielectric permittivity $\varepsilon^{*}=\varepsilon^{\prime}-i \varepsilon^{\prime \prime}$ of GPI along the $b$-direction at several frequencies are shown in Fig. $1 \mathrm{a}$ and $\mathrm{b}$. The dielectric permittivity at $100 \mathrm{~Hz}$ frequency reaches 3500 in a peak at $T_{\mathrm{c}}=223.6 \mathrm{~K}$. It is the highest value obtained for this crystal indicating a good quality of samples used in the current work. The dielectric permittivity fulfills the Curie-Weiss law with the Curie constant $C_{\mathrm{p}}=375 \mathrm{~K}$ in the paraelectric phase in temperature region of about $20 \mathrm{~K}$ above the Curie point. The constant $C_{\mathrm{p}}$ is much smaller than that for another hydrogen bonded crystal of BPI (betaine phosphite) containing phosphite anions chains $[9,10]$.

The ferroelectric dispersion in the vicinity of $T_{\mathrm{c}}$ begins at about a few $\mathrm{MHz}$ and ranges up to the millimeter waves. The maximum value of $\varepsilon^{\prime}=\varepsilon_{\mathrm{c}}$ under clamped adiabatic conditions at $90 \mathrm{MHz}$ is about 450 . This value is close to that of another glycine containing ferroelectric diglycine nitrate [11]. The minimum of $\varepsilon^{\prime}$ observed at $T_{\mathrm{c}}$ indicates critical slowing down of polarization fluctuations. The ferroelectric dispersion near $T_{\mathrm{c}}$ is almost over at $27 \mathrm{GHz}$, where $\varepsilon_{\infty} \approx 6.4$. In the paraelectric phase dielectric relaxation is described by Cole-Cole formula [12] with a single relaxation time $\tau$ ( $\beta=1$; Debye-type relaxation)

$$
\varepsilon^{*}=\varepsilon_{\infty}+\frac{\varepsilon_{c}-\varepsilon_{\infty}}{1+(\mathrm{i} \omega \tau)^{\beta}} .
$$

Cole-Cole plots for GPI are shown in Fig. 2a and $b$ for the paraelectric and ferroelectric phases, respectively. The relaxation time of correlated dipoles motion 

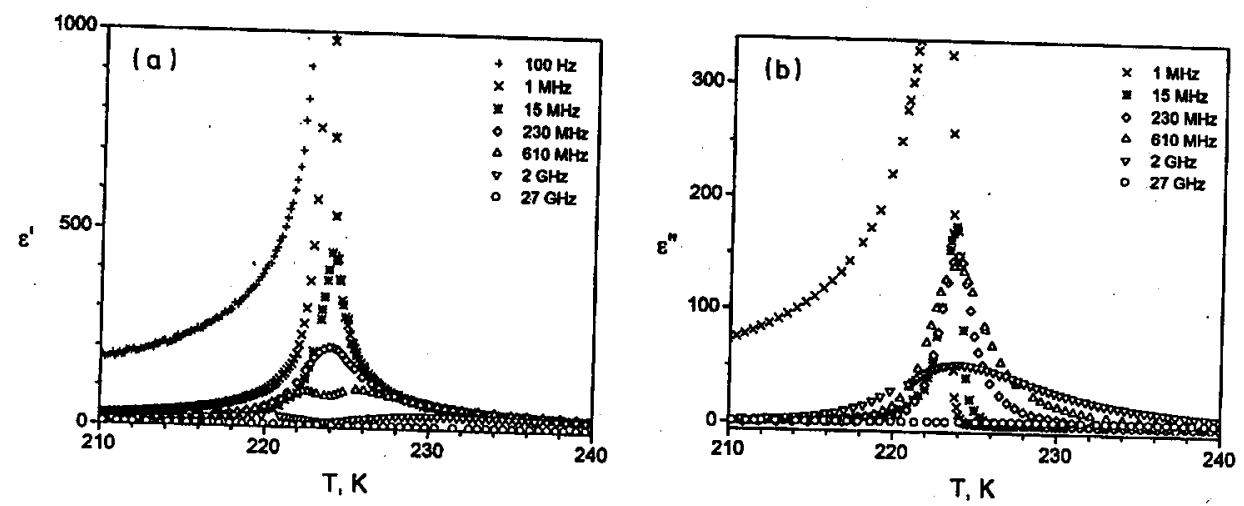

Fig. 1. Temperature dependences of (a) $\varepsilon^{\prime}$ and (b) $\varepsilon^{\prime \prime}$ for GPI crystal along the $b$-axis for various lrequencies.
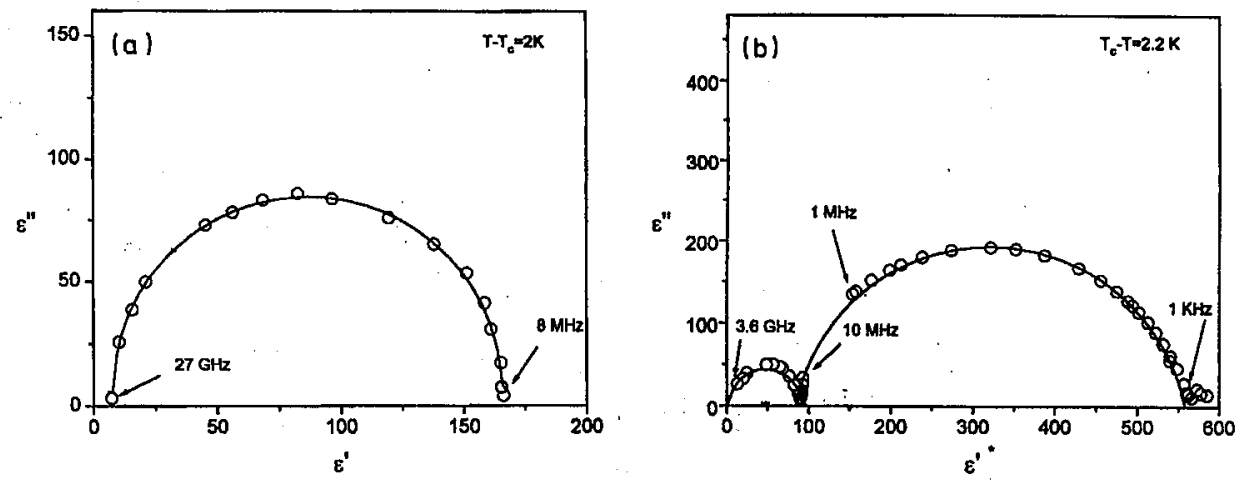

Fig. 2. Cole-Cole plots for GPI crystal (a) in paraelectric phase and (b) in ferroelectric phase.

was calculated according to the results presented in Fig. 1 by a least squares fit. The spectrum of $\varepsilon^{\prime \prime}(f)$ obtained from the experiment is well described by Cole-Cole formula in the paraelectric phase as it is shown in Fig. 3 and confirms Debye-type relaxation $(\beta=1)$. The relaxation frequency fulfills the Curie-Weiss law $f_{\mathrm{s}}=(2 \pi \tau)^{-1}=0.305\left(T-T_{\mathrm{c}}\right) \mathrm{GHz}$ in the paraelectric phase (Fig. 4).

Near the Curie point the temperature dependence of the relaxation time in GPI saturates and reaches a value of $\tau=5.4 \times 10^{-10} \mathrm{~s}$. This can be caused by inhomogeileities, e.g. surface layers and spatial variations of the Curie temperature.

Since at $100 \mathrm{MHz}$ the domain contribution to the permittivity is suppressed the dielectric permittivity under clamped adiabatic conditions in the ferroelectric phase fulfills the Curie-Weiss law with $C_{\mathrm{f}}=154 \mathrm{~K}$. The ratio $C_{\mathrm{p}} / C_{\mathrm{f}}=2.4$ is rather close to the second order phase transition (see Fig. 4). At $1 \mathrm{MHz}$ domain wall contribution to permittivity still persists in the ferroelectric phase and the Curie-Weiss constant is of the same value as at $1 \mathrm{MHz}$ and $90 \mathrm{MHz}$ in the paraelectric one (see Fig. 4). Taking into account the symmetry of GPI we applied 

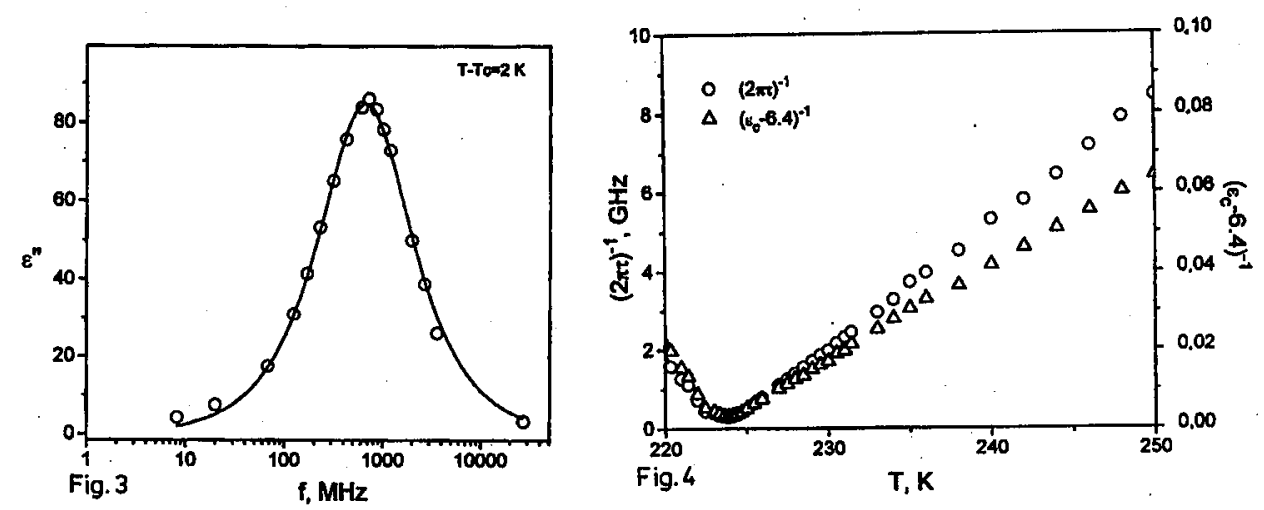

Fig. 3. Frequency dependence of $\varepsilon^{\prime \prime}$ for GPI crystal at $T-T_{c}=2 \mathrm{~K}$.

Fig. 4. Temperature dependences of frequency $f_{\mathrm{s}}=(2 \pi \tau)^{-1}$, reciprocal dielectric contribution of relaxational ferroelectric mode $(100 \mathrm{MHz})$ and the Curie-Weiss law at $1 \mathrm{MHz}$.

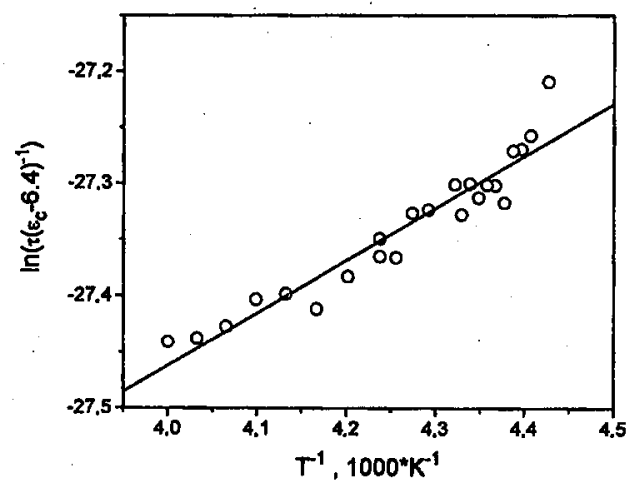

Fig. 5. Dependence of $\ln \left(\tau\left(\varepsilon_{\mathrm{c}}-\varepsilon_{\infty}\right)^{-1}\right)$ versus $1000 / T$ for GPI crystal.

Mason's model of ferroelectricity for crystal, where paraelectric phase is not piezoelectric [13]. In this model asymmetrical double minimum potential for the motion of the electrical dipoles, which are responsible for the polarization, is assumed. In this case the relaxation time in the paraelectric phase varies according to

$$
\tau=\tau_{0}\left(\varepsilon_{\mathrm{c}}-\varepsilon_{\infty}\right) \exp (\Delta U / k T)
$$

where $\left(2 \pi \tau_{0}\right)^{-1}=f_{0}$ is the constant frequency factor and $\Delta U$ is the activation energy for the electrical dipoles. In Fig. 5 the reciprocal of the kinetic coefficient, $\tau\left(\varepsilon_{\mathrm{c}}-\varepsilon_{\infty}\right)^{-1}$ is plotted against $1 / T$. We obtained the values $\Delta U=(0.0401 \pm$ $0.0027) \mathrm{eV}$ and $\tau_{0}=(1.840 \pm 0.006) \times 10^{-13} \mathrm{~s}$ (for $T>T_{\mathrm{c}}$ ). The magnitude of the activation energy is larger than $k T_{\mathrm{c}}\left(\Delta U=(2.07 \pm 0.13) k T_{\mathrm{c}}\right)$. Thus, the order-disorder type of phase transition in GPI crystal is confirmed.

As it is seen in Fig. $2 b$ two well-distributed relaxational processes could be distinguished in the ferroelectric phase. The high frequency relaxational process is of the same nature as in the paraelectric phase (ferroelectric dipolar dispersion). 

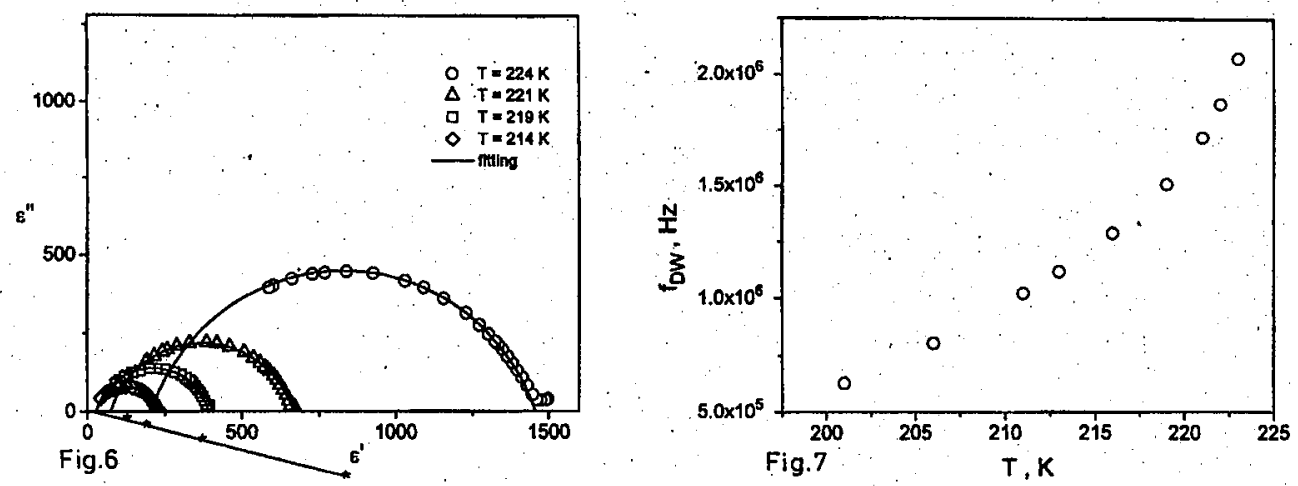

Fig. 6. Cole-Cole plots for low frequency relaxational process in the ferroelectric phase of GPI crystal.

Fig. 7. Temperature dependence of low frequency relaxational process for GPI crystal.

The second process (observed only below $T_{\mathrm{c}}$ ) is most probably related to domain structure appearance and domain wall motion. Large contribution to the permittivity observed at low frequencies $(100 \mathrm{~Hz}, 1 \mathrm{kHz})$ is suppressed at $90 \mathrm{MHz}$. The Cole-Cole diagrams for some chosen temperatures connected with the second relaxational process are shown in Fig. 6. The dispersion in the temperature range down to $215 \mathrm{~K}$ is described by the formula (1), where $\beta$ coefficient is equal to $0.82 \pm 0.01$. The temperature dependence of low frequency $f_{\mathrm{DW}}$ is shown in Fig. 7 . Below $215 \mathrm{~K}$ the dispersion is not Cole-Cole type-like any more.

To elucidate the mechanism of the low frequency dispersion more detailed studies under the influence of dc electric field are now in progress.

The dynamical properties obtained in the range $T_{\mathrm{c}}+25 \mathrm{~K}$ are very close to those observed in the vicinity of $T_{\mathrm{c}}$ [5]. It means that relaxation in GPI crystal is connected with the same dipole in a very broad temperature range (about $25 \mathrm{~K}$ above $T_{c}$ ) in the paraelectric phase.

On the ground of the results presented one can conclude:

1. a GPI crystal undergoes a continuous phase transition of the order-disorder type;

2. dynamical dielectric properties in the paraelectric phase are described in terms of electrical dipoles flipping in double potential well with activation energy $\Delta U=(2.07 \pm 0.13) k T_{\mathrm{c}}$; the origin of electric dipoles along the $b$-axis is most probably related to proton motion along the symmetrical double well hydrogen bonds;

3. pronounced low-frequency dispersion related to domain walls dynamics is observed in ferroelectric phase of GPI.

This work was supported by the Committee for Scientific Research 2016/W/IFD/1996.

\section{References}

[1] S. Dacko, Z. Czapla, J. Baran, M. Drozd, Phys. Lett. A 223, 217 (1996).

[2] M.-T. Averbuch-Pouchot, Acta Crystallogr. C 49, 815 (1993). 
[3] G.E. Bacon, R.S. Pease, Proc. R. Soc. Lond. A 220, 397 (1953).

[4] G.E. Bacon, R.S. Pease, Proc. R. Soc. Lond. A 230, 359 (1955).

[5] J. Baran, G. Bator, R. Jakubas, M. Śledż, J. Phys., Condens. Matter 8, 10647 (1996).

[6] J. Baran, M. Śledź, R. Jakubas, G. Bator, Phys. Rev. B 55, 169 (1997).

[7] A. Brilingas, R. Davidovitch, J. Grigas, S. Lapinskas, M. Medkov, V. Samulionis, V. Skritskii, Phys. Status Solidi A 96, 101 (1986).

[8] J. Grigas, A. Brilingas, V. Kalesinskas, Ferroelectrics 107, 61 (1990).

[9] J. Albers, A. Klöpperpieper, H.J. Rother, S. Haussühl, Ferroelectrics 81, 27 (1988).

[10] R. Sobiestianskas, J. Grigas, Z. Czapla, S. Dacko, Phys. Status Solidi A 136, 223 (1993).

[11] R. Sobiestianskas, J. Grigas, Z. Czapla, Ferroelectrics 100, 187 (1989).

[12] K.S. Cole, R.H. Cole, J. Chem. Phys. 9, 341 (1941).

[13] W.P. Mason, Piezoelectric Crystals and their Application to Ultrasonic, Van Nostrand Co., New York 1950. 